\title{
ORTHOGRAPHIC REFLECTANCE IMAGE FOR PLANAR TARGET LOCALIZATION IN LOW DENSITY TLS POINT CLOUDS
}

\author{
Dongxu Guo ${ }^{\mathrm{a}, \mathrm{b}}$, Dapeng Yu ${ }^{\mathrm{c}}$, Yubin Liang ${ }^{\mathrm{a}, \mathrm{b}, *}$, Chenyang Feng ${ }^{\mathrm{a}, \mathrm{b}}$ \\ ${ }^{a}$ School of Geographic and Environmental Sciences, Tianjin Normal University, Tianjin 300387, China \\ ${ }^{\mathrm{b}}$ Tianjin Engineering Center for Geospatial Information Technology, Tianjin Normal University, Tianjin 300387, China \\ ${ }^{\mathrm{c}}$ Shen Kan Engineering \& Technology Corporation, MCC, Shenyang 110169, China \\ 1015881693@qq.com; 108518769@qq.com; lyb.whu@gmail.com; 1447100725@qq.com
}

\section{WG II/3}

KEY WORDS: Terrestrial Laser Scanning, Point Cloud, Target Localization, Point Density, Orthographic Reflectance Image, Point Cloud Registration

\begin{abstract}
:
Point cloud registration is important and essential task for terrestrial laser scanning applications. Point clouds acquired at different positions exhibit significant variation in point density. Most registration methods implicitly assume dense and uniform distributed point clouds, which is hardly the case in large-scale surveying. The accuracy and robustness of feature extraction are greatly influenced by the point density, which undermines the feature-based registration methods. We show that the accuracy and robustness of target localization dramatically decline with decreasing point density. A methodology for localization of artificial planar targets in low density point clouds is presented. An orthographic image of the target is firstly generated and the potential position of the target center is interactively selected. Then the 3D position of the target center is estimated by a non-linear least squares adjustment. The presented methodology enables millimeter level accuracy of target localization in point clouds with $30 \mathrm{~mm}$ sample interval. The robustness and effectiveness of the methodology is demonstrated by the experimental results.
\end{abstract}

\section{INTRODUCTION}

Terrestrial laser scanning is an effective technique for threedimensional data acquisition of indoor and outdoor environment in a short period of time. It has been widely used in civil engineering, archaeology, and 3D reconstruction of industrial facilities (Sansoni et al., 2009; Rusu et al., 2008). In order to obtain relatively complete information of the environment, laser scans acquired at different positions have to be registered. In the past years researchers have proposed many registration methods which can be roughly separated to iterative and non-iterative ones. Iterative registration methods start from an initial state and iteratively register two point clouds until converge. One of the earliest published iterative registration methods is the Iterative Closest Point (ICP) method (Besl and McKay, 1992). ICP is commonly used for register point clouds of small-scale objects such as sculptures, cultural relics and industrial facilities (Levoy et al., 2000). Much improvement has been made on the correspondence search strategy and variants of the ICP have been used to register point clouds of large scale environments (Yang and Medioni, 1992; Rusinkiewicz and Levoy, 2001; Bae and Lichti, 2008; Gressin et al., 2013).

Non-iterative methods directly solve the transformation parameters between two point clouds using geometric features. Geometric features may come from the scanned environments or the preinstalled artificial targets (Rabbani et al., 2007; Franaszek et al., 2009). Features can be extracted and localized directly in the point clouds (Rusu et al., 2009; Theiler et al.,
2014). Features can also be extracted and localized in the images generated from the point clouds (Kang et al., 2009; Liang et al., 2017; Weinmann et al., 2011).

Most registration methods implicitly assume dense and uniform distributed point clouds, which is hardly the case in terrestrial laser scanning applications. The point density of the scanned object is dependent on its distance to the scanner, the direction of its surface relative to the scanner and the angular resolution of the scanner. Point clouds acquired at different scan positions exhibit significant variation in point density.

Features in a point cloud fade with decreasing point density. It has been shown that planar features estimated using scanning points with less point density are less precise (Soudarissanane et al., 2011). As will also be shown in the experimental results of this paper, the accuracy and robustness of feature extraction and localization dramatically decline with decreasing point density, which undermines the feature-based registration methods.

The standard approach to alleviate the problems of varying point density is to re-sample the point clouds in a preprocessing step. However, such re-sampling process has several shortcomings. First, detailed features may be discarded, leading to smoothing of the point cloud. Second, the sample interval needs to be set for each specific scenario as it depends on the geometry and scale of the point cloud.

\footnotetext{
* Corresponding author
} 
Point cloud registration using artificial targets is usually more accurate and reliable. Researchers and practitioners often preinstall artificial targets (e.g. marker points, positioning sphere and planar targets) around research objects and use centers of the targets as correspondences to register point clouds afterwards. Planar paper target is widely used because of its low cost and convenience. A typical paper target and its point cloud are shown in Figure 1.
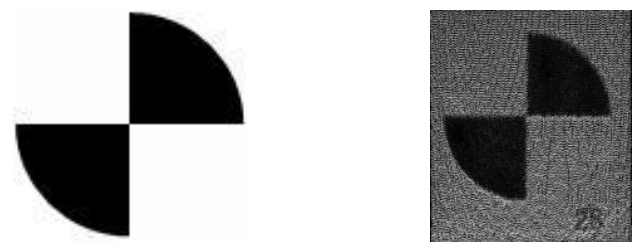

Figure 1. A typical black/white target and its point cloud

Although decreasing point density makes it difficult to extract the targets, the intensities/reflectance of the scanning points still exhibits salient visual pattern that can be used to recognize and localize the artificial targets. In this paper, we present a methodology for localization of artificial planar targets in low density point clouds. The methodology is detailed in the following section. The experimental results are discussed in section 3 and conclusion is made in section 4.

\section{METHODOLOGY}

The presented methodology consists of two major procedures. Orthographic images of targets are firstly generated and the potential position of the target center is interactively selected. Then the $3 \mathrm{D}$ positions of the target centers are estimated by a non-linear least squares adjustment.

To generate an orthographic image of a target, Principal Component Analysis (PCA) is used to extract eigen values and eigen vectors of covariance matrix derived from the spatial coordinates of the target. Assume the extracted eigen values are $\lambda_{0} \geq \lambda_{1} \geq \lambda_{2}$ and the corresponding eigen vectors are $v_{0}$, $v_{1}$ and $v_{2}$. The plane of the target can be expressed as the linear combination of $v_{0}$ and $v_{1}$.

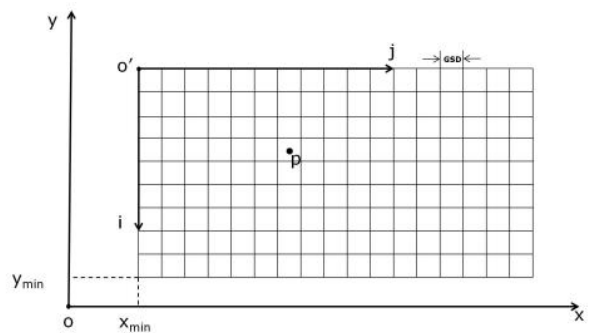

Figure 2. The projection coordinate system and pixel coordinate system

To rasterize an orthographic image, the projection coordinates should be transformed to the pixel coordinates and the gray values should be evaluated. The relationship between the projection coordinate system oxy and the pixel coordinates $o^{\prime} i j$ is shown in Figure 2. Given the projection coordinates $(x, y)$ of a point $p$ and the ground sample distance GSD of the orthographic image, the corresponding pixel coordinates $(i, j)$ of $p$ can be derived from equation (1).

$$
\left\{\begin{array}{l}
i=\operatorname{nrow}-\operatorname{round}\left(\left(y-y_{\min }\right) / G S D\right) \\
j=\operatorname{round}\left(\left(x-x_{\min }\right) / G S D\right)
\end{array}\right.
$$

Where $x_{\min }$ and $y_{\min }$ are the minimums of all the projection coordinates. nrow is the number of rows of the orthographic image. Gray values of the orthographic image can be obtained from reflectance values of the scanning points. To enhance the contrast of the orthoimage, the reflectance values are linear stretched to the range $[0,255]$.

To register point clouds using targets, the three dimensional coordinates of target centers should be determined. The orthoimage exhibits salient pattern of the target, which is easy to select the target center. If the selected center point lies at the projection of a laser point, its three dimensional coordinates can be directly obtained. Otherwise, its spatial coordinates are estimated as follows.

The spatial coordinates of the target center and one of its neighboring points can be related using the three-dimensional Euclidean distance equation as

$$
d=\sqrt{\left(X_{i}-X_{c}\right)^{2}+\left(Y_{i}-Y_{c}\right)^{2}+\left(Z_{i}-Z_{c}\right)^{2}}
$$

Where $d$ is the distance between the target center and one of its neighboring points. The coordinates $\left(X_{C}, Y_{C}, Z_{C}\right)^{T}$ of the target center are the unknowns. $\left(X_{i}, Y_{i}, Z_{i}\right)^{T}$ are the coordinates of a neighboring point. Function $d$ can be expanded at a given point $\left(X_{0}, Y_{0}, Z_{0}\right)^{T}$ using Taylor series with the first order derivatives as

$$
d \approx d_{0}+\left(\frac{\partial d}{\partial X_{C}}\right)_{0} v_{X_{C}}+\left(\frac{\partial d}{\partial Y_{C}}\right)_{0} v_{Y_{C}}+\left(\frac{\partial d}{\partial Z_{C}}\right)_{0} v_{Z_{C}}
$$

Where $d_{0}$ is the value of function $d$ evaluated at the given point $\left(X_{0}, Y_{0}, Z_{0}\right)^{T} .\left(v_{X_{C}}, v_{Y_{C}}, v_{Z_{C}}\right)^{T}$ is the incremental vector of the unknowns $\left(X_{C}, Y_{C}, Z_{C}\right)^{T}$. And the partial derivatives in equation (3) can be calculated by

To assess the influence of image downsampling, the aerial images are downsampled using the free and open-source software ImageMagick. Then image matching and image orientation resulted from the original and the downsampled images are compared.

$$
\left(\frac{\partial d}{\partial X_{c}}\right)_{0}=\frac{X_{C}-X_{i}}{\sqrt{\left(X_{i}-X_{C}\right)^{2}+\left(Y_{i}-Y_{C}\right)^{2}+\left(Z_{i}-Z_{C}\right)^{2}}}
$$




$$
\begin{aligned}
& \left(\frac{\partial d}{\partial Y_{c}}\right)_{0}=\frac{Y_{C}-Y_{i}}{\sqrt{\left(X_{i}-X_{C}\right)^{2}+\left(Y_{i}-Y_{C}\right)^{2}+\left(Z_{i}-Z_{C}\right)^{2}}} \\
& \left(\frac{\partial d}{\partial Z_{c}}\right)_{0}=\frac{Z_{C}-Z_{i}}{\sqrt{\left(X_{i}-X_{C}\right)^{2}+\left(Y_{i}-Y_{C}\right)^{2}+\left(Z_{i}-Z_{C}\right)^{2}}}
\end{aligned}
$$

And ()$_{0}$ indicates that the partial derivatives are evaluated at the given point $\left(X_{0}, Y_{0}, Z_{0}\right)^{T}$.With $\mathrm{n}$ observations (spatial coordinates of the neighboring points), the normal matrix can be expressed as

$$
A^{T} A V=A^{T} W
$$

Where

$$
\begin{aligned}
A_{n \times 3} & =\left[\begin{array}{ccc}
\frac{\partial d_{1}}{\partial X_{c}} & \frac{\partial d_{1}}{\partial Y_{c}} & \frac{\partial d_{1}}{\partial Z_{c}} \\
\vdots & \vdots & \vdots \\
\frac{\partial d_{i}}{\partial X_{c}} & \frac{\partial d_{i}}{\partial Y_{c}} & \frac{\partial d_{i}}{\partial Z_{c}} \\
\vdots & \vdots & \vdots \\
\frac{\partial d_{n}}{\partial X_{c}} & \frac{\partial d_{n}}{\partial Y_{c}} & \frac{\partial d_{n}}{\partial Z_{c}}
\end{array}\right]_{0}^{T} \\
V & =\left[\begin{array}{ccc}
v_{X_{c}} & v_{Y_{c}} & v_{Z_{c}}
\end{array}\right]^{T} \\
W_{n \times 1} & =\left[\begin{array}{c}
d_{1}-d_{1}^{0} \\
\vdots \\
d_{i}-d_{i}^{0} \\
\vdots \\
d_{n}-d_{n}^{0}
\end{array}\right]
\end{aligned}
$$

Where $A_{n \times 3}$ is the Jacobian matrix. The vector $\left(d_{1}^{0}, \cdots, d_{n}^{0}\right)^{T}$ in $W$ are the distances evaluated at the given point $\left(X_{0}, Y_{0}, Z_{0}\right)^{T}$. The vector $\left(d_{1}, \cdots, d_{n}\right)^{T}$ in $W$ are the observed distances between the target center and the neighboring points. When more than three neighboring points are observed, $V$ can be solved by equation (11).

$$
V=\left(A^{T} A\right)^{-1} A^{T} W
$$

The unknowns $\left(X_{C}, Y_{C}, Z_{C}\right)^{T}$ can be updated using $V$ and finally determined in an iterative manner. The target localization procedure is described in Algorithm 1.

\section{Algorithm 1}

Input: The point cloud of a planar target $P$. The ground sample distance GSD of the orthographic reflectance image. The convergence condition $T$ and the number of iterations threshold $N$.

$$
x y=p c a(X Y Z)
$$

$$
\begin{aligned}
& \text { sref }=\text { linstretch }(\text { ref }) \\
& \text { ori }=\text { rasterize }(x y, G S D, \text { sref })
\end{aligned}
$$

interactively select the target center in the ori initialize $\left(X_{C}, Y_{C}, Z_{C}\right)^{T}$ and $V$

initialize the number of iterations niter

while niter $<=N$ and $\left(\operatorname{abs}\left(v_{X_{C}}\right)>T\right.$ or $\operatorname{abs}\left(v_{Y_{C}}\right)>$

$\left.T \operatorname{or} \operatorname{abs}\left(v_{Z_{C}}\right)>T\right)$

calculate the Jacobian matrix $A$ and vector $W$

solve $V$

update $\left(X_{C}, Y_{C}, Z_{C}\right)^{T}=\left(X_{C}+V_{X_{C}}, Y_{C}+V_{Y_{C}}, Z_{C}+V_{Z_{C}}\right)^{T}$

$$
\text { niter }++
$$

\section{end while}

Output: The spatial coordinates $\left(X_{C}, Y_{C}, Z_{C}\right)^{T}$ of the target center.

The input of the procedure includes the point cloud $P$ of the target, the ground sample distance GSD of the orthographic reflectance image, the convergence condition $T$ and the number of iterations threshold $N$. $P$ consists of the spatial coordinates $X Y Z$ and the reflectance ref of the scanning points. Function $p c a$ is used to derive the projection coordinates $x y$ of the neighboring points from their spatial coordinates $X Y Z$. The reflectance ref is linearly stretched to sref using linstretch function. The planar coordinates $x y$ and the sref is then used to rasterize the orthographic reflectance image ori.

After interactively select the potential position of the target center in ori, $\left(X_{C}, Y_{C}, Z_{C}\right)^{T}$ is iteratively solved using the Gauss-Newton method. The $\left(X_{C}, Y_{C}, Z_{C}\right)^{T}$ can be initialized to the average of the coordinates of its neighboring points. The distance between two $3 \mathrm{D}$ points is preserved under orthographic projection, which can be seen from Figure 3. Therefore, the observed distances $\left(d_{1}, \cdots, d_{n}\right)^{T}$ can be derived from the image points of the target center and the neighboring points in the orthographic reflectance image.

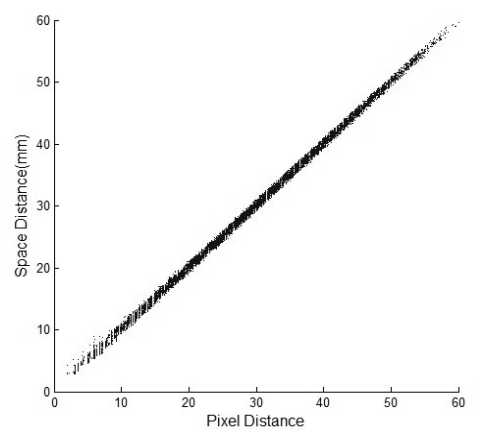

Figure 3. Relationship between space distance and pixel distance 
The procedure converges when the absolute values of $v_{X_{C}}$, $v_{Y_{C}}$ and $v_{Z_{C}}$ are less than or equal to the convergence condition $T$ or the number of iterations niter is larger than the threshold $N$.

\section{RESULTS AND DISCUSSION}

To demonstrate the effectiveness of the presented method, a test field is setup (Figure 4). 30 targets are uniformly preinstalled on the wall. The test field is scanned with a RIEGL VZ-1000 laser scanner. The vertical and horizontal resolution of the scanning is set to 0.03 degrees and $39,671,920$ points are acquired.
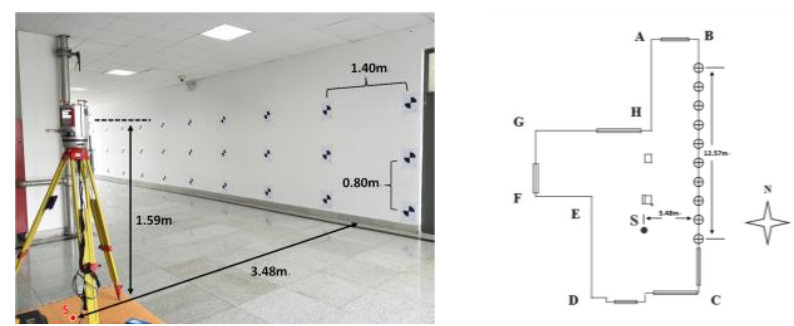

Figure 4. The setup of the test field and the floor plan

To the convenience of the point cloud processing, the point cloud of the targets is cropped (Figure 5). All of the 30 targets are then localized using Leica Cyclone 6.0.3 and used as the ground truth.

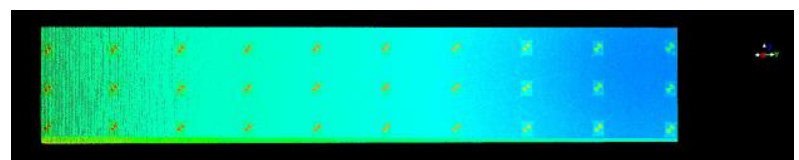

Figure 5. The cropped point cloud of the targets

In order to evaluate the influence of the point density on the accuracy and robustness of target localization, we decimate the point cloud via 3D gridding with $5 \mathrm{~mm}$-step sample intervals. The targets in the decimated point clouds are localized using Cyclone. Coordinates of the localized targets from the decimated point clouds are compared with those from the original point cloud. It is worth mentioning that each of the targets has been localized several times and the coordinates of the visually best localized one are kept. If one target cannot be localized after several tries, it is considered as non-localizable. The target localization accuracy using Cyclone is shown in Table 2.

Table 1. The target localization accuracy using Cyclone

\begin{tabular}{|c|c|c|c|}
\hline $\begin{array}{c}\text { Sample } \\
\text { Interval } \\
(\mathbf{m m})\end{array}$ & Accuracy (m) & Max Absolute & $\begin{array}{c}\text { Number of } \\
\text { Localizable } \\
\text { Targets }\end{array}$ \\
\hline $\mathbf{5}$ & $(0.003,0.001,0.001)$ & $(0.013,0.004,0.002)$ & 30 \\
\hline $\mathbf{1 0}$ & $(0.002,0.001,0.001)$ & $(0.008,0.002,0.002)$ & 30 \\
\hline
\end{tabular}

\begin{tabular}{|c|c|c|c|}
\hline $\mathbf{1 5}$ & $(0.004,0.001,0.003)$ & $(0.011,0.003,0.010)$ & 30 \\
\hline $\mathbf{2 0}$ & $(0.007,0.002,0.004)$ & $(0.017,0.006,0.011)$ & 28 \\
\hline $\mathbf{2 5}$ & $(0.008,0.004,0.010)$ & $(0.017,0.010,0.026)$ & 19 \\
\hline
\end{tabular}

The Accuracy column of Table 1 lists the Root Mean Square Error (RMSE) along each dimension. It is easy to find that the accuracy of the target localization along $\mathrm{X}, \mathrm{Y}$ and $\mathrm{Z}$ axis decreases with increasing sample intervals, which can also be seen from Figure 6. Table 1 also shows that the maximum absolute error increases with increasing sample intervals.

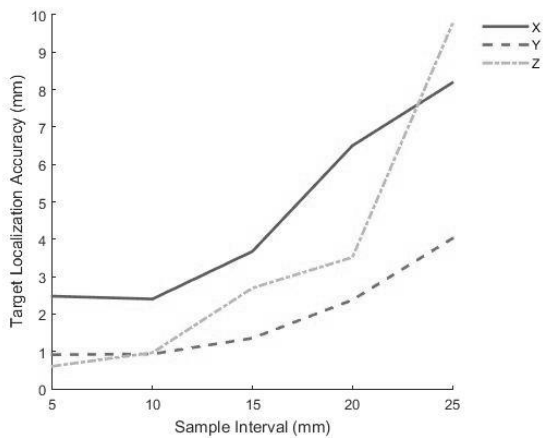

Figure 6. Relationship between the sample intervals and the accuracy of target localization using Cyclone

Figure 7 shows that the number of the localizable targets drops dramatically with increasing sample intervals. When the sample interval approaches $20 \mathrm{~mm}$, some targets becomes nonlocalizable. Only two targets are localizable when the sample interval equals $30 \mathrm{~mm}$. When the sample interval exceeds $35 \mathrm{~mm}$, none of the targets are localizable.

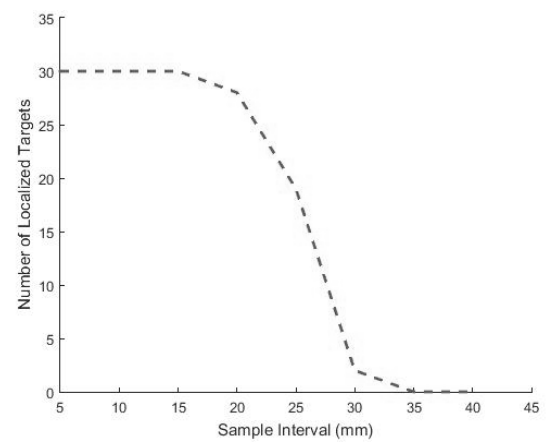

Figure 7. Relationship between the sample intervals and the number of the localizable targets

The presented method is implemented using Matlab. The point cloud of each target is used as the input of the program. The ground sample distance of the orthoimage is set to $2 \mathrm{~mm}$ and the background color is set to white. Figure 8 shows the generated orthoimages of a typical target from point clouds with different sample intervals. 


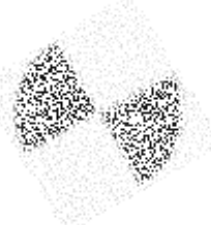

(a)

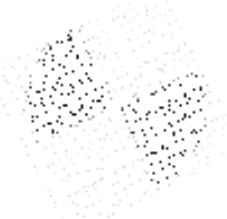

(b)

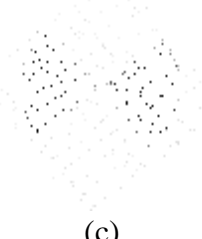

(c)

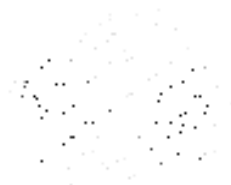

(d)

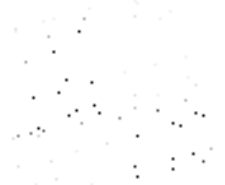

(e)

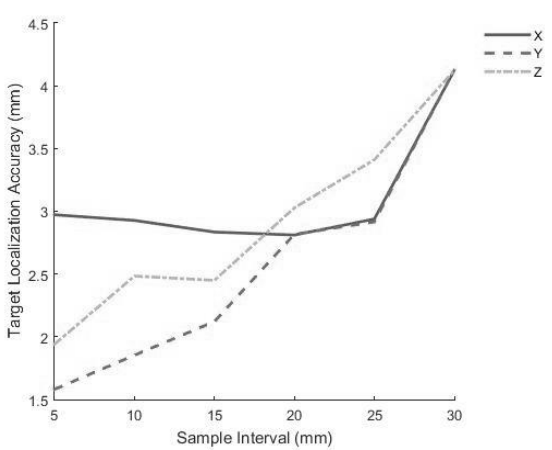

Figure 9. Relationship between the sample intervals and the accuracy of target localization using the presented method

In comparison with Cyclone, the presented method can localize all the targets when the sample interval exceeds $20 \mathrm{~mm}$ In the experiments, all the targets are still localizable using the presented method when the sample interval equals $30 \mathrm{~mm}$, and millimeter localization accuracy is achieved. The experimental results show that the presented method is more robust and accurate than Cyclone for target localization in low density point clouds.

\section{CONCLUSIONS AND FUTURE WORK} least squares manner as detailed in section 2. In the experiment, the convergence condition is set to $2 \mathrm{~mm}$ and the number of iterations threshold is set to 20. The target localization accuracy is shown in Table 2.

Table 2 . The target localization accuracy using the presented method

\begin{tabular}{|c|c|c|c|}
\hline $\begin{array}{c}\text { Sample } \\
\text { Interval (mm) }\end{array}$ & Accuracy (m) & $\begin{array}{c}\text { Max Absolute Error } \\
\text { (m) }\end{array}$ & $\begin{array}{c}\text { Number of } \\
\text { Localizable } \\
\text { Targets }\end{array}$ \\
\hline 5 & $(0.003,0.002,0.002)$ & $(0.007,0.003,0.007)$ & 30 \\
\hline 10 & $(0.003,0.002,0.003)$ & $(0.006,0.004,0.006)$ & 30 \\
\hline 15 & $(0.003,0.002,0.002)$ & $(0.006,0.005,0.005)$ & 30 \\
\hline 20 & $(0.003,0.003,0.003)$ & $(0.005,0.006,0.006)$ & 30 \\
\hline 25 & $(0.003,0.003,0.003)$ & $(0.006,0.008,0.007)$ & 30 \\
\hline 30 & $(0.0040 .003,0.004)$ & $(0.008,0.007,0.008)$ & 30 \\
\hline
\end{tabular}

It is easy to find that the accuracy of the target localization along $\mathrm{X}, \mathrm{Y}$ and $\mathrm{Z}$ axis decreases while the sample interval increases, which can be seen from Figure 9. Table 2 shows that the target localization accuracy of the presented method is comparable to that of Cyclone when the sample interval is within $15 \mathrm{~mm}$. However, when the sample interval exceeds $20 \mathrm{~mm}$, the accuracy of the presented method is higher than that of Cyclone. Table 2 also shows that the maximum absolute error is smaller than that of Cyclone when the sample interval exceeds $20 \mathrm{~mm}$.
Point density greatly influences the accuracy and robustness of target localization in point cloud processing. A methodology for localization of planar targets in low density TLS point clouds is presented in this paper. Orthoimages of targets are firstly generated and the potential position of the target center is interactively selected. The 3D positions of the target centers are estimated by a non-linear least squares adjustment. The experimental results show that the presented method is robust and accurate for target localization in low density point clouds. The presented method can also be used to locate other features in low density point clouds. An alternative way to improve the target localization accuracy in low density point cloud is to increase the point density, which will be examined in our future work.

\section{REFERENCES}

Bae, K.-H., Lichti, D.D., 2008. A method for automated registration of unorganised point clouds. ISPRS-J. Photogramm. Remote Sens. 63, 36-54.

Besl, P.J., McKay, H.D., 1992. A method for registration of 3D shapes. IEEE Trans. Pattern Anal. Mach. Intell. 14, 239-256.

Franaszek, M., Cheok, G.S., Witzgall, C., 2009. Fast automatic registration of range images from 3D imaging systems using sphere targets. Automation in Construction 18, 265-274.

Gressin, A., Mallet, C., Demantké, J., David, N., 2013. Towards 3D lidar point cloud registration improvement using optimal neighborhood knowledge. ISPRS-J. Photogramm. Remote Sens. 79, 240-251.

Kang, Z., Li, J., Zhang, L., Zhao, Q., Zlatanova, S., 2009. Automatic Registration of Terrestrial Laser Scanning Point Clouds using Panoramic Reflectance Images. Sensors (Basel, Switzerland) 9, 2621-2646. 
Levoy, M., Pulli, K., Curless, B., Rusinkiewicz, S., Koller, D., Pereira, L., Ginzton, M., Anderson, S., Davis, J., Ginsberg, J., Shade, J., Fulk, D., 2000. The digital Michelangelo project: 3D scanning of large statues, Proceedings of the 27th annual conference on Computer graphics and interactive techniques. ACM Press/Addison-Wesley Publishing Co., pp. 131-144.

Liang, Y.B., Qiu, Y., Cui, T.J., 2017. Semiautomatic Registration of Terrestrial Laser Scanning Data Using Perspective Intensity Images. IEEE Geoscience and Remote Sensing Letters 14, 28-32.

Rabbani, T., Dijkman, S., van den Heuvel, F., Vosselman, G., 2007. An integrated approach for modelling and global registration of point clouds. ISPRS-J. Photogramm. Remote Sens. 61, 355-370.

Rusinkiewicz, S., Levoy, M., 2001. Efficient Variants of the ICP Algorithm, 3DIM. IEEE Computer Society, pp. 145-152.

Rusu, R.B., Blodow, N., Beetz, M., 2009. Fast Point Feature Histograms (FPFH) for 3D registration, Robotics and Automation, 2009. ICRA '09. IEEE International Conference on, pp. 3212-3217.

Rusu, R.B., Marton, Z.C., Blodow, N., Dolha, M., Beetz, M., 2008. Towards 3D Point cloud based object maps for household environments. Robot. Auton. Syst. 56, 927-941.

Sansoni, G., Trebeschi, M., Docchio, F., 2009. State-of-TheArt and Applications of 3D Imaging Sensors in Industry, Cultural Heritage, Medicine, and Criminal Investigation. Sensors 9, 568-601.

Soudarissanane, S., Lindenbergh, R., Menenti, M., Teunissen, P., 2011. Scanning geometry: Influencing factor on the quality of terrestrial laser scanning points. ISPRS-J. Photogramm. Remote Sens. 66, 389-399.

Theiler, P.W., Wegner, J.D., Schindler, K., 2014. Keypointbased 4-Points Congruent Sets - Automated marker-less registration of laser scans. ISPRS-J. Photogramm. Remote Sens. 96, 149-163.

Weinmann, M., Weinmann, M., Hinz, S., Jutzi, B., 2011. Fast and automatic image-based registration of TLS data. ISPRS-J. Photogramm. Remote Sens. 66, S62-S70.

Yang, C., Medioni, G., 1992. Object modelling by registration of multiple range images. Image and Vision Computing 10, 145-155. 